\title{
Stems and spectral sequences
}

\author{
HANS JOACHIM BAUES \\ DAVID BLANC
}

\begin{abstract}
We introduce the category $\mathscr{P}_{\text {ste }} m[n]$ of $n$-stems, with a functor $\mathcal{P}[n]$ from spaces to $\mathscr{P}_{\text {ste }}[n]$. This can be thought of as the $n$-th order homotopy groups of a space. We show how to associate to each simplicial $n$-stem $\mathcal{Q}_{\bullet}$ an $(n+1)$-truncated spectral sequence. Moreover, if $\mathcal{Q}_{\bullet}=\mathcal{P}[n] X_{\bullet}$ is the Postnikov $n$-stem of a simplicial space $X_{\bullet}$, the truncated spectral sequence for $\mathcal{Q}_{\bullet}$ is the truncation of the usual homotopy spectral sequence of $X_{\bullet}$. Similar results are also proven for cosimplicial $n$-stems. They are helpful for computations, since $n$-stems in low degrees have good algebraic models.
\end{abstract}

55T05; 18G40, 18G55, 55S45, 55T15, 18G30, 18G10

\section{Introduction}

Many of the spectral sequences of algebraic topology arise as the homotopy spectral sequence of a (co)simplicial space - including the spectral sequence of a double complex, the (stable or unstable) Adams spectral sequence, the Eilenberg-Moore spectral sequence, and so on (see Examples 5.14). Given a simplicial space $X_{\bullet}$, the $E^{2}$-term of its homotopy spectral sequence has the form $E_{s, t}^{2}=\pi_{s} \pi_{t} X_{\bullet}$, so it may be computed by applying the homotopy group functor dimensionwise to $X_{\bullet}$.

In this paper we show that the higher terms of this spectral sequence are obtained analogously by applying "higher homotopy group" functors to $X_{\bullet}$. These functors are given explicitly in the form of certain Postnikov stems, defined in Section 2; the Postnikov 0 -stem of a space is equivalent to its homotopy groups.

We then show how the $E^{r}$-term of the homotopy spectral sequence of a simplicial space $X_{\bullet}$ can be described in terms of the $(r-2)$-Postnikov stem of $X_{\bullet}$, for each $r \geq 2$ (see Theorem 4.13) - and similarly for the homotopy spectral sequence of a cosimplicial space $X^{\bullet}$ (see Theorem 5.12).

As an application for the present paper, in [5] we generalize the first author's result with Mamuka Jibladze in [6], which shows that the $E^{3}$-term of the stable Adams spectral sequence can be identified as a certain secondary derived functor Ext. We do this by 
showing how to define in general the higher order derived functors of a continuous functor $F: \mathcal{C} \rightarrow \mathcal{T}_{*}$, by applying $F$ to a simplicial resolution $W_{\bullet}$ in $\mathcal{C}$, and taking Postnikov $n$-stems of $F W_{\bullet}$.

1.1 Notation and conventions The category of pointed connected topological spaces will be denoted by $\mathcal{T}_{*}$; that of pointed sets by $\mathscr{S}_{e t_{*}}$; that of groups by $\mathscr{G}_{p}$. For any category $\mathcal{C}, s \mathcal{C}$ denotes the category of simplicial objects over $\mathcal{C}$, and $c \mathcal{C}$ that of cosimplicial objects over $\mathcal{C}$. We abbreviate $s \mathscr{S} e t$ to $\mathcal{S}, s \mathscr{S}_{e t} t_{*}$ to $\mathcal{S}_{*}$, and $s \mathscr{G}_{p}$ to $\mathcal{G}$. For any small indexing category $I$, the category of functors $I \rightarrow \mathcal{C}$ is denoted by $\mathcal{C}^{I}$.

Acknowledgements We wish to thank the referee for his or her careful reading of the paper and helpful comments on it.

\section{Postnikov stems}

The Postnikov system of a topological space (or simplicial set) $X$ is the tower of fibrations

$$
\cdots \rightarrow P^{n+1} X \stackrel{p^{n+1}}{\longrightarrow} P^{n} X \stackrel{p^{n}}{\longrightarrow} P^{n-1} X \cdots P^{1} X \stackrel{p^{1}}{\longrightarrow} P^{0} X,
$$

equipped with maps $q^{n}: X \rightarrow P^{n} X$ (with $p^{n} \circ q^{n}=q^{n-1}$ ), which induce isomorphisms on homotopy groups in degrees $\leq n$. Here $P^{n} X$ is $n$-coconnected (that is, $\pi_{i} P^{n} X=0$ for $i>n)$ and $\pi_{i} p^{n}$ is an isomorphism for $i<n$. The fiber of the map $p^{n}: P^{n} X \rightarrow$ $P^{n-1} X$ is the Eilenberg-Mac Lane space $K\left(\pi_{n} X, n\right)$, so the fibers are determined up to homotopy by $\pi_{*} X$. Thus a generalization of the homotopy groups of $X$ is provided by the following notion:

2.2 Definition For any $n \geq 0$, a Postnikov $n-$ stem in $\mathcal{T}_{*}$ is a tower

$$
\mathcal{Q}:=\left(\cdots \rightarrow Q_{k+1} \stackrel{q_{k+1}}{\longrightarrow} Q_{k} \stackrel{q_{k}}{\longrightarrow} Q_{k-1} \cdots Q_{0}\right)
$$

in $\mathcal{T}_{*}^{(\mathbb{N}, \leq)}$, in which $Q_{k}$ is $(k-1)$-connected and $(n+k)$-coconnected (so that $\pi_{i}\left(Q_{k}\right)=0$ for $i<k$ or $\left.i>n+k\right)$ and $\pi_{i}\left(q_{k}\right)$ is an isomorphism for $k \leq i<n+k$. Here $(\mathbb{N}, \leq)$ is the usual linearly ordered category of the natural numbers. The space $Q_{k}$ is called the $k$-th $n$-window of $\mathcal{Q}$.

Such an $n$-stem is thus a collection of overlapping ( $k-1)$-connected $n+k$-types, which may be depicted for $n=2$ as follows:

$$
\begin{aligned}
& \text {...*** } \\
& \text { * * * } \\
& \text { * * * } \\
& \text { * } * * \ldots
\end{aligned}
$$


where each row exhibits the $n+1$ nontrivial homotopy groups (denoted by $*$ ) of one $n$-window, and all those in the $i$-th column (corresponding to $\pi_{i}$ ) are isomorphic.

We denote by $\mathscr{P}_{\text {stem }}[n]$ the full subcategory of Postnikov $n$-stems in the functor category $\mathcal{T}_{*}^{(\mathbb{N}, \leq)}$ (with model category structure on the latter as in Hirschhorn [20, 11.6]). Thus the morphisms in $\mathscr{P}_{s t e m}[n]$ are given by strictly commuting maps of towers, and $f: \mathcal{Q} \rightarrow \mathcal{Q}^{\prime}$ is a weak equivalence (respectively, a fibration) if each $f_{k}: Q_{k} \rightarrow Q_{k}^{\prime}$ is such. This lets us define the homotopy category of Postnikov $n$-stems, ho $\mathscr{P}_{\text {stem }}[n]$, as a full subcategory of ho $\mathcal{T}_{*}^{(\mathbb{N}, \leq)}$.

The category $\mathscr{P}_{\text {stem }}[n]$ is pointed, has products, and is equipped with canonical functors

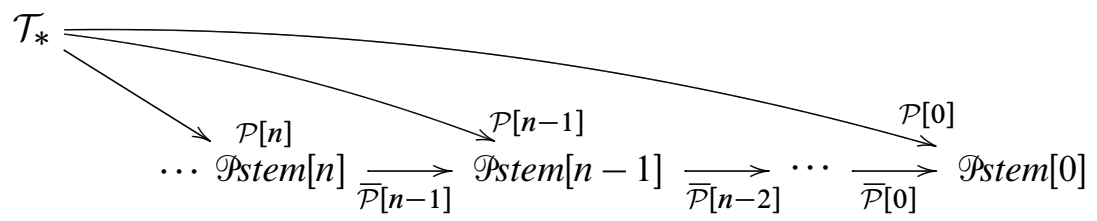

which preserve products and weak equivalences.

2.5 Remark The sequence of functors (2.6) is described by a commuting diagram, in which we may take all maps to be fibrations:

(2.6)

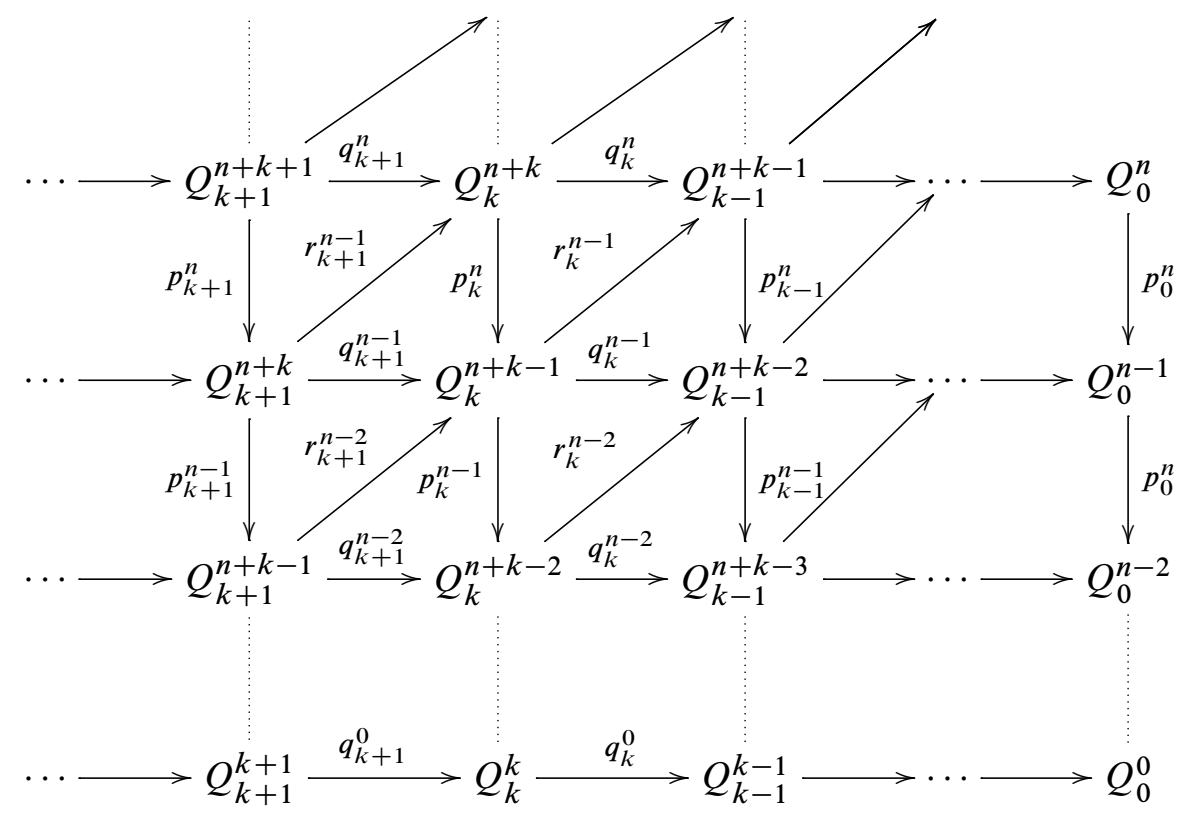


Here $\pi_{i} Q_{k}^{n}=0$ for $i<k$ or $i>n$, and all maps induce isomorphisms in $\pi_{i}$ whenever possible. Thus:

(a) The $k$-th column (from the right) is the Postnikov tower for $Q_{k}:=\lim _{n} Q_{k}^{n}$.

(b) The diagonals are the dual Postnikov system of connected covers for $Q_{0}^{j}$.

(c) The $n$-th row (from the bottom) is a Postnikov $n$-stem.

(d) In particular, each space in the 0-stem (the bottom row) is an EilenbergMac Lane space, and the maps $q_{k}^{0}$ are nullhomotopic. Thus the homotopy type of the bottom line in ho $\mathscr{P}_{s t e m}[0]$ is determined by the collection of homotopy groups $\left\{\pi_{k} Q_{k}^{k}\right\}_{k=0}^{\infty}$.

2.7 Definition The motivating example of a Postnikov $n$-stem is a realizable one, associated to a space $X \in \mathcal{T}_{*}$, and denoted by $\mathcal{P}[n] X$, with $(P[n] X)_{k}:=P^{n+k} X\langle k\rangle$. As usual, $Y\langle k\rangle$ denotes the $(k-1)$-connected cover of a space $Y \in \mathcal{T}_{*}$. Each fibration $q_{k}:(P[n] X)_{k} \rightarrow(P[n] X)_{k-1}$ fits into a commuting triangle of fibrations

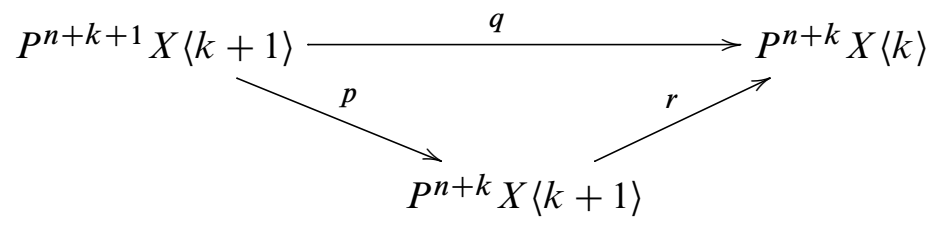

in which the maps $p$ and $r$ are the fibration of (2.1) and the covering map, respectively. See our paper [4, Section 10.5] for a natural context in which nonrealizable Postnikov $n$-stems arise.

2.9 Examples of stems The functor $\mathcal{P}[0]_{*}: \mathcal{T}_{*} \rightarrow$ ho $\mathscr{P}_{\text {stem }}[0]$ induced by $\mathcal{P}[0]$ is equivalent to the homotopy group functor: in fact, the homotopy groups of a space define a functor $\pi_{*}: \mathcal{T}_{*} \rightarrow \mathcal{K}$ into the product category $\mathcal{K}:=\prod_{i=0}^{\infty} \mathcal{K}_{i}$, where $\mathcal{K}_{0}=\mathscr{Y}_{\text {et }}$, $\mathcal{K}_{1}=\varphi_{p}$, and $\mathcal{K}_{i}=A_{b} \varphi_{p}$, for $i \geq 2$. Moreover, there is an equivalence of categories $\vartheta: \mathcal{K} \equiv$ ho $\mathscr{P}_{\text {stem }}[0]$, such that the functor $\mathcal{P}[0]_{*}$ is equivalent to the composite functor $\vartheta \circ \pi_{*}: \mathcal{T}_{*} \rightarrow \mathcal{K}$.

Similarly, the functor $\mathcal{T}_{*} \rightarrow$ ho $\mathscr{P}_{\text {stem }}[1]$ induced by $\mathcal{P}$ [1] is equivalent to the secondary homotopy group functor of the first author and Muro [7, Section 4], in the sense that each secondary homotopy group $\pi_{n, *} X$ completely determines the $n$-th 1 -window of $X$. Using the results on secondary homotopy groups in [7], one obtains a homotopy

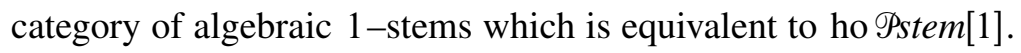

A category of algebraic models for 2-stems is only partially known. The homotopy classification of $(k-1)$-connected $(k+2)$-types is described for all $k$ in [3]; this theory can be used to classify homotopy types of Postnikov 2-stems. 


\section{The spectral sequence of a simplicial space}

We begin with the construction of the homotopy spectral sequence for a simplicial space (cf Quillen [21], Bousfield and Friedlander [14, Theorem B.5], and Bousfield and Kan [15, X, Section 6]), using the version given by Dwyer, Kan and Stover in [19, Section 8] (see also Bousfield [11; 12, Section 2,5] and Dwyer, Kan and Stover [18, Section 3.6]). For this purpose, we require some explicit constructions for the $E^{2}-$ model category of simplicial spaces.

3.1 Definition Given a simplicial object $X_{\bullet} \in s \mathcal{C}$, over a complete pointed category $\mathcal{C}$, for each $n \geq 1$ define its $n$-cycles object to be

$$
Z_{n} X_{\bullet}:=\left\{x \in X_{n} \mid d_{i} x=* \text { for } i=0, \ldots, n\right\} .
$$

Similarly, the $n$-chains object for $X \bullet$ is

$$
C_{n} X_{\bullet}:=\left\{x \in X_{n} \mid d_{i} x=* \text { for } i=1, \ldots, n\right\} .
$$

Set $Z_{0} X_{\bullet}:=X_{0}$. We denote the map $\left.d_{0}\right|_{C_{n} X_{\bullet}}: C_{n} X_{\bullet} \rightarrow Z_{n-1} X_{\bullet}$ by $\mathbf{d}_{0}^{X_{n}}$.

3.2 Notation For any nonnegatively graded object $T_{*}$, we write $\Omega T_{*}$ for the graded object with $\left(\Omega T_{*}\right)_{j}:=T_{j+1}$ for all $j \geq 0$. The notation is motivated by the natural isomorphism of graded groups $\pi_{*} \Omega X \cong \Omega\left(\pi_{*} X\right)$ for $X \in \mathcal{T}_{*}$.

3.3 Definition Now assume that $\mathcal{C}$ is a pointed model category of spaces, such as $\mathcal{T}_{*}$ or $\mathcal{G}$, and $X_{\bullet}$ is a Reedy fibrant simplicial object over $\mathcal{C}$ - that is, for each $n \geq 1$, the universal face map $\delta_{n}: X_{n} \rightarrow M_{n} X_{\bullet}$ into the $n$-th matching object of $X_{\bullet}$. is a fibration (see [20,15.3]). The map $\mathbf{d}_{0}=\mathbf{d}_{0}^{X_{n}}$ then fits into a fibration sequence in $\mathcal{C}$ :

$$
\cdots \Omega Z_{n} X_{\bullet} \rightarrow Z_{n+1} X_{\bullet} \stackrel{j_{n+1}^{X}}{\longrightarrow} C_{n+1} X_{\bullet} \stackrel{\mathbf{d}_{0}^{X_{n+1}}}{\longrightarrow} Z_{n} X_{\bullet}
$$

(see [19, Proposition 5.7]).

For each $n \geq 0$, the $n$-th natural homotopy group of the simplicial space $X_{\bullet}$, denoted by $\pi_{n}^{\natural} X_{\bullet}=\pi_{n, *}^{\natural} X_{\bullet}$, the cokernel of the map $\left(\mathbf{d}_{0}^{X_{n+1}}\right)_{\#}$ (induced on homotopy groups by $\mathbf{d}_{0}^{X_{n+1}}$ ). Note that the cokernel of a maps of groups or pointed sets is generally just a pointed set.

We thus have an exact sequence of graded groups:

$$
\pi_{*} C_{n+1} X_{\bullet} \stackrel{\left(\mathbf{d}_{0}^{X} X_{n+1}\right)_{\#}}{\longrightarrow} \pi_{*} Z_{n} X_{\bullet} \stackrel{\widehat{\vartheta}_{n}}{\longrightarrow} \pi_{n, *}^{\natural} X_{\bullet} \rightarrow 0 .
$$

Together the groups $\left(\pi_{n, k}^{\natural} X_{\bullet}\right)_{n, k=0}^{\infty}$ constitute the bigraded homotopy groups of [19, Section 5.1]. 
3.6 Construction of the spiral sequence Applying the functor $\pi_{*}$ to the fibration sequence (3.4) yields a long exact sequence, with connecting homomorphism $\partial_{\#}: \Omega \pi_{*} Z_{n} X_{\bullet}=\pi_{*} \Omega Z_{n} X_{\bullet} \rightarrow \pi_{*} Z_{n+1} X_{\bullet}$. Note that the inclusion $\iota: C_{n} X_{\bullet} \hookrightarrow X_{n}$ induces an isomorphism $\iota_{\star}: \pi_{*} C_{n} X_{\bullet} \cong C_{n}\left(\pi_{*} X_{\bullet}\right)$ for each $n \geq 0$ (see [10, Proposition 2.7]). From (3.5) we see that

$$
\Omega \pi_{n}^{\natural} X_{\bullet}=\Omega \operatorname{Coker}\left(\mathbf{d}_{0}^{X_{n+1}}\right)_{\#} \cong \operatorname{Im} \partial_{\#} \cong \operatorname{Ker}\left(j_{n+1}^{X \bullet}\right)_{\#} \subseteq \pi_{*} Z_{n+1} X_{\bullet},
$$

so we obtain a commutative diagram with exact rows and columns

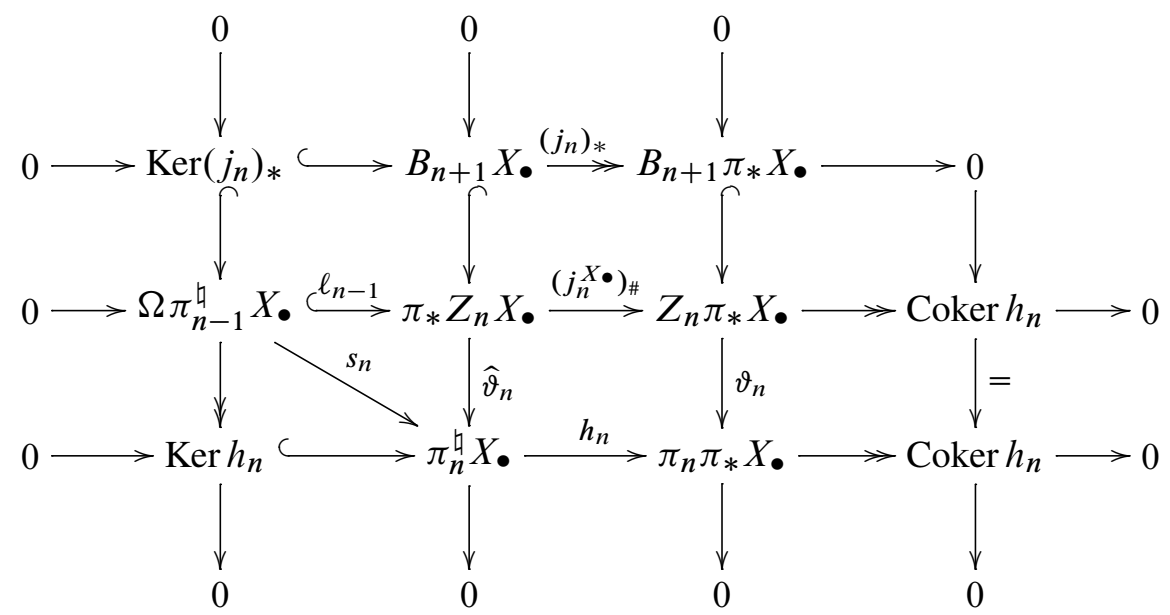

in which $B_{n+1} X_{\bullet}:=\operatorname{Im}\left(\mathbf{d}_{0}^{X_{n+2}}\right)_{\#} \subseteq \pi_{*} Z_{n} X_{\bullet}$ and $B_{n+1} \pi_{*} X_{n+2}:=\operatorname{Im} \mathbf{d}_{0}^{\pi_{*} X_{n+2}}$ are the respective boundary objects. Note that the map

$$
\left(j_{n}^{X \bullet}\right)_{\#}: \pi_{*} Z_{n} X_{\bullet} \rightarrow \pi_{*} C_{n} X_{\bullet}
$$

induced by the inclusion $j_{n}^{X_{\bullet}}$ of (3.4) above in fact factors through $Z_{n} \pi_{*} X_{\bullet}$, as indicated in the middle row of (3.7).

This defines the map of graded groups $h_{n}: \pi_{n}^{\natural} X_{\bullet} \rightarrow \pi_{n}\left(\pi_{*} X_{\bullet}\right)$. Note that for $n=0$ the map $\hat{l}_{\star}$ is an isomorphism, so $h_{0}$ is, too. The map

$$
s_{n}: \Omega \pi_{n-1}^{\natural} X_{\bullet} \rightarrow \pi_{n}^{\natural} X \bullet
$$

is the composite of the inclusion

$$
\ell_{n-1}: \operatorname{Ker}\left(j_{n}^{X \bullet}\right)_{\#} \hookrightarrow \pi_{*} Z_{n} X \bullet
$$

with the quotient map

$$
\hat{\vartheta}_{n}: \pi_{*} Z_{n} X_{\bullet} \rightarrow \pi_{n}^{\natural} X_{\bullet}
$$

of (3.5), using the natural identification of $\Omega \pi_{n}^{\natural} X_{\bullet}$ with $\operatorname{Ker}\left(j_{n+1}^{X_{\bullet}}\right)_{\#}$. 
The map $\partial_{n+2}: \pi_{n+2} \pi_{*} X_{\bullet} \rightarrow \Omega \pi_{n}^{\natural} X_{\bullet}$ is induced by the composite

$$
Z_{n+2} \pi_{*} X_{\bullet} \subseteq C_{n+2} \pi_{*} X_{\bullet} \cong \pi_{*} C_{n+2} X_{\bullet} \stackrel{\left(\mathbf{d}_{0}^{\left.X_{n+2}\right)_{\#}} \longrightarrow\right.}{\longrightarrow} \pi_{*} Z_{n+1} X_{\bullet},
$$

which actually lands in $\operatorname{Ker}\left(j_{n+1}^{X_{\bullet}}\right)_{\#}$ by the exactness of the long exact sequence for the fibration (3.4).

These maps $s_{n}, h_{n}$, and $\partial_{n}$ fit into a spiral long exact sequence:

$$
\begin{aligned}
\cdots \rightarrow \Omega \pi_{n-1}^{\natural} X \bullet & \stackrel{s_{n}}{\longrightarrow} \pi_{n}^{\natural} X \bullet \stackrel{h_{n}}{\longrightarrow} \pi_{n} \pi_{*} X \bullet \stackrel{\partial_{n}}{\longrightarrow} \Omega \pi_{n-2}^{\natural} X_{\bullet} \\
& \stackrel{s_{n-1}}{\longrightarrow} \pi_{n-1}^{\natural} X_{\bullet} \rightarrow \cdots \rightarrow \pi_{0}^{\natural} X \bullet \stackrel{\cong}{\rightarrow} \pi_{0} \pi_{*} X_{\bullet}
\end{aligned}
$$

$(\operatorname{cf}[19,8.1])$

3.10 The spectral sequence of a simplicial space For any simplicial space $X_{\bullet} \in s \mathcal{T}_{*}$ (or bisimplicial set), Bousfield and Friedlander showed that there is a first-quadrant spectral sequence of the form

$$
E_{s, t}^{2}=\pi_{s} \pi_{t} X_{\bullet} \Rightarrow \pi_{s+t}\left\|X_{\bullet}\right\|,
$$

where $\left\|X_{\bullet}\right\| \in \mathcal{T}_{*}$ is the realization (or the diagonal, in the case of $X_{\bullet} \in s \mathcal{S}_{*}$ ). The spectral sequence is always defined, but $X_{\bullet}$. must satisfy certain "Kan conditions" to guarantee convergence - see [14, Theorem B.5].

In [19, Section 8.4], Dwyer, Kan and Stover showed that (3.11) coincides up to sign, from the $E^{2}$-term on, with the spectral sequence associated to the exact couple of (3.4), which we call the spiral spectral sequence for $X_{\bullet}$.

If we assume that each $X_{n}$ is connected, by taking loops (or applying Kan's functor $G$, if $X_{\bullet} \in s \mathcal{S}_{*}$ ), we may replace $X_{\bullet}$ by a bisimplicial group $G X_{\bullet} \in s \mathcal{G}$, and then (3.11) becomes the spectral sequence of [21].

\section{Simplicial stems and truncated spectral sequences}

As noted in Section 2.9, the $E^{2}$-term of any of the above equivalent spectral sequences for a simplicial space $X_{\bullet}$ is determined explicitly by the simplicial 0 -stem of $X_{\bullet}$.

Our goal is to extend this description to the higher terms of the spectral sequence. For this purpose, fix $n \geq 0$, and consider a simplicial Postnikov $n$-stem $\mathcal{Q}$. (which need not be realizable as $\mathcal{P}[n] X_{\bullet}$ for some simplicial space $\left.X_{\bullet}\right)$. This is equivalent to having a collection of simplicial spaces $\mathcal{Q}_{\bullet}^{n+k}\langle k\rangle$ for each $k \geq 0$, equipped with maps as in (2.3), with $\pi_{i} \mathcal{Q}_{\bullet}^{n+k}\langle k\rangle=0$ for $i<k$ or $i>n+k$. 
We assume that $\mathcal{Q} \bullet$ is Reedy fibrant in the sense that for each $k \geq 0$, the simplicial space $\mathcal{Q}_{\bullet}^{n+k}\langle k\rangle$ is Reedy fibrant. In this case, the " $n$-stem version" of the spiral long exact sequence is defined as follows: for each $t, i, k \geq 0$, set

$$
\begin{aligned}
\pi_{t, i}^{\natural(k, n)} \mathcal{Q}_{\bullet} & :=\pi_{t, i+k}^{\natural} \mathcal{Q}_{\bullet}^{n+k}\langle k\rangle, \\
\pi_{i}^{(k, n)} \mathcal{Q}_{\bullet}: & =\pi_{i+k} \mathcal{Q}_{\bullet}^{n+k}\langle k\rangle= \begin{cases}\pi_{i+k} \mathcal{Q}_{\bullet} & \text { if } 0 \leq i \leq n, \\
0 & \text { otherwise. }\end{cases}
\end{aligned}
$$

Note that the $(i+k)$-th homotopy group $\pi_{i+k} \mathcal{Q} \bullet$ of a Postnikov $n$-stem $\mathcal{Q} \bullet$ is well-defined, and coincides with $\pi_{i+k} X_{\bullet}$ for $0 \leq i \leq n$ when $\mathcal{Q}_{\bullet}=\mathcal{P}[n] X_{\bullet}$.

4.2 Definition The collection of long exact sequences (3.9) for $\mathcal{Q}_{\bullet}^{n+k}\langle k\rangle$ (indexed by $k \geq 0)$ :

$$
\cdots \Omega \pi_{t-1, *}^{\natural(k, n)} \mathcal{Q} \bullet \stackrel{s_{t}^{(k, n)}}{\longrightarrow} \pi_{t, *}^{\natural(k, n)} \mathcal{Q} \bullet \stackrel{h_{t}^{(k, n)}}{\longrightarrow} \pi_{t} \pi_{*}^{(k, n)} \mathcal{Q}_{\bullet} \stackrel{\partial_{t}^{(k, n)}}{\longrightarrow} \Omega \pi_{t-2, *}^{\natural(k, n)} \mathcal{Q}_{\bullet} \cdots,
$$

together with the maps between adjacent $k$-windows induced by the map $q$ in (2.6), will be called the spiral $n$-system of $\mathcal{Q}_{\bullet}$. When $\mathcal{Q}_{\bullet}=\mathcal{P}[n] X_{\bullet}$, we will refer to this simply as the spiral $n$-system of $X_{\bullet}$.

4.4 Remark Using the exactness of (4.3), definition (4.1) implies that

$$
\pi_{t, i}^{\natural(k, n)} \mathcal{Q}_{\bullet}=\pi_{t, i}^{\natural} \mathcal{Q}_{\bullet}^{n+k}\langle k\rangle=0 \text { for } i>n,
$$

by induction on $t \geq 0$. Note, however, that while the groups $\pi_{i}^{(k, n)} \mathcal{Q}_{\bullet}$ are explicitly described by (4.1), the dependence of $\pi_{t, i}^{\natural(k, n)} \mathcal{Q}_{\bullet}$ on $k$ and $n$ requires more care.

4.6 The $E^{2}$-term of the spectral sequence The spiral 0 -system of a simplicial Postnikov $0-$ stem $\mathcal{Q} \bullet$ reduces to a series of isomorphisms

$$
h_{t}: \pi_{t, *}^{\natural(k, 0)} \mathcal{Q}_{\bullet} \cong \pi_{t} \pi_{*}^{(k, 0)} \mathcal{Q}_{\bullet}
$$

(for each $k \geq 0$ ). When $\mathcal{Q}_{\bullet}=\mathcal{P}[0] X_{\bullet}$ is the Postnikov 0 -stem of a simplicial space $X_{\bullet}$, this allows us to identify the $E_{t, k}^{2}$-term of the spiral spectral sequence for $X_{\bullet}$, which is $\pi_{t} \pi_{k} X_{\bullet}=\pi_{t} \pi_{k} P^{0+k} X_{\bullet}\langle k\rangle=\pi_{t} \pi_{k}\left(P[0] X_{\bullet}\right)_{k}=\pi_{t} \pi_{*}^{(k, 0)} \mathcal{P}[0] X_{\bullet}=\pi_{t} \pi_{*}^{(k, 0)} \mathcal{Q}_{\bullet}$, with $\pi_{t, *}^{\natural(k, 0)} \mathcal{Q}_{\bullet}=\pi_{t, *}^{\natural(k, 0)} \mathcal{P}[0] X_{\bullet}$.

The first interesting case is the spiral 1-system, for which we have: 
4.7 Proposition The $E^{3}$-term of the spiral spectral sequence for a simplicial space $X$. is determined by the spiral 1 -system of $X_{\bullet}$. In fact, $d_{t, k}^{2}$ may be identified with

$$
\partial_{t}^{(k, 1)}: \pi_{t} \pi_{k} X_{\bullet} \rightarrow \Omega \pi_{t-2,0}^{\natural(k, 1)} X_{\bullet},
$$

while $E_{t, k}^{3}$ is the image of the composite map

$$
\begin{aligned}
\pi_{t, 0}^{\natural(k, 1)} X_{\bullet} \stackrel{h_{t}^{(k, 1)}}{\longrightarrow} \pi_{t} \pi_{k} X \bullet \cong \pi_{t} \pi_{1}^{(k-1,1)} X_{\bullet} & \\
& \stackrel{h_{t}^{(k-1,1)}}{\cong} \pi_{t, 1}^{\natural(k-1,1)} X_{\bullet} \stackrel{s_{t+1}^{(k-1,1)}}{\longrightarrow} \pi_{t+1,0}^{\natural(k-1,1)} X_{\bullet} .
\end{aligned}
$$

Observe that (4.8) involves maps from different windows of the spiral 1-system, implicitly identified using the isomorphisms induced by the map $q$ in (2.6).

Proof Because $n=1$ throughout, we abbreviate $\pi_{t, i}^{\natural(k, 1)} \mathcal{Q} \bullet$ to $\pi_{t, i}^{\natural(k)} \mathcal{Q} \bullet$ and $\pi_{i}^{(k, 1)} \mathcal{Q} \bullet$ to $\pi_{i}^{(k)} \mathcal{Q} \bullet$ observing that $\pi_{i}^{(k)} \mathcal{Q} \bullet$ is simply $\pi_{i+k} X_{\bullet}$ for $i=0$, 1 , and zero otherwise, since $\mathcal{Q}_{\bullet}=\mathcal{P}[1] X_{\bullet}$. Thus the spiral $1-\operatorname{system}(4.3)$ is nontrivial for each $t \geq 1$ in (internal) degrees $i=0,1$ only, and we can write it in two rows:

$$
0 \longrightarrow \pi_{t, 1}^{\natural(k)} \mathcal{Q} \bullet \stackrel{\cong}{\longrightarrow} \pi_{t} \pi_{1}^{(k)} \mathcal{Q} \bullet \longrightarrow 0 \longrightarrow \pi_{t-1,1}^{\natural(k)} \mathcal{Q} \bullet \stackrel{\cong}{\longrightarrow} \pi_{t-1} \pi_{1}^{(k)} \mathcal{Q} \bullet
$$

$\Omega \pi_{t-1,0}^{\natural(k)} \mathcal{Q} \bullet \stackrel{s_{t}}{\rightarrow} \pi_{t, 0}^{\natural(k)} \mathcal{Q} \bullet \stackrel{h_{t}}{\rightarrow} \pi_{t} \pi_{0}^{(k)} \mathcal{Q} \bullet \stackrel{\partial_{t}}{\rightarrow} \Omega \pi_{t-2,0}^{\natural(k)} \mathcal{Q} \bullet$

$$
\stackrel{s_{t-1}}{\longrightarrow} \pi_{t-1,0}^{\natural(k)} \mathcal{Q} \bullet \stackrel{h_{t-1}}{\longrightarrow} \pi_{t-1} \pi_{0}^{(k)} \mathcal{Q} \bullet
$$

Since $\mathcal{Q}_{\bullet}:=\mathcal{P}[1] X_{\bullet}$ is the simplicial Postnikov 1 -stem of $X_{\bullet}$, we actually have a collection of two-row long exact sequences, one for each $k$-window of $\mathcal{P}[1] X_{\bullet}$.

For each such $k$-window $\mathcal{P}_{k}[1] X_{\bullet}$, we can use the top row to identify

$$
\Omega \pi_{t, 0}^{\natural(k)} \mathcal{Q} \bullet=\Omega \pi_{t, 0}^{\natural} \mathcal{P}_{k}[1] X_{\bullet}=\pi_{t, 1}^{\natural} \mathcal{P}_{k}[1] X_{\bullet}=\pi_{t, 1}^{\natural(k)} \mathcal{Q} \bullet
$$

with

$$
\pi_{t} \pi_{1}^{(k)} \mathcal{Q}_{\bullet}=\pi_{t} \pi_{t}^{(1)} \mathcal{P}_{k}[1] X_{\bullet}=\pi_{t} \pi_{k+1} X_{\bullet},
$$

so the bottom row reduces to:

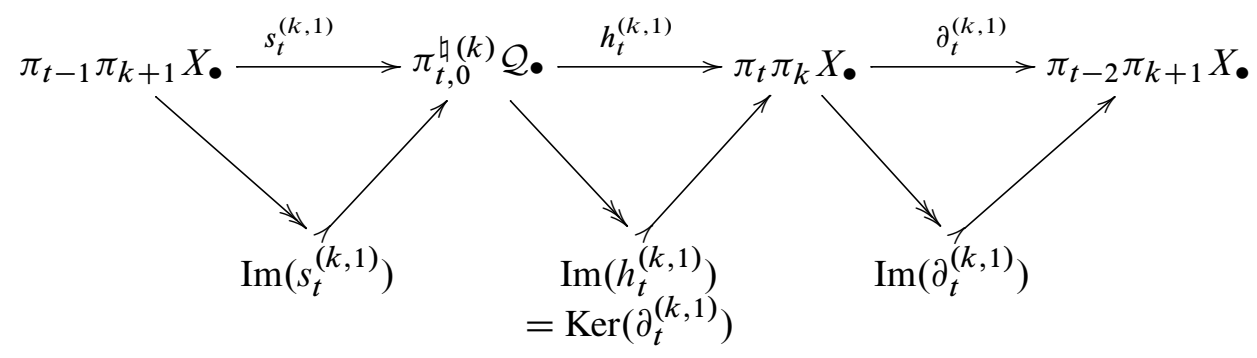


Note that the following part of the $E^{1}$-term of the exact couple for the fibration sequence $C_{n+1} P^{1} \Omega^{i} X_{\bullet} \rightarrow Z_{n} P^{1} \Omega^{i} X_{\bullet}$, (as in (3.4)):

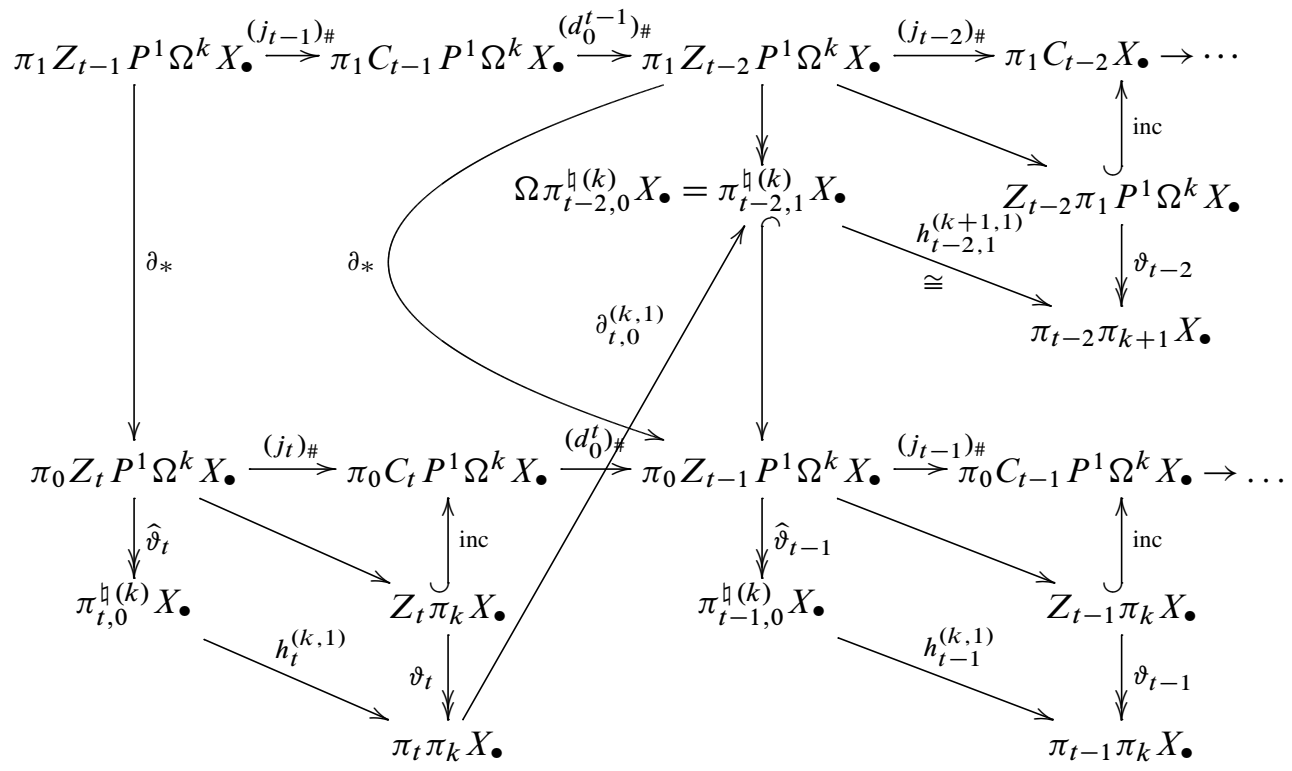

is naturally isomorphic to the exact couple for $C_{n+1} \Omega^{k} X_{\bullet} \rightarrow Z_{n} \Omega^{k} X_{\bullet}$, since $C_{n+1}$ and $Z_{n}$ are limits, so they commute with $P^{1}$, and then

$$
\pi_{1} P^{1} Z_{t-1} \Omega^{k} X_{\bullet} \cong \pi_{1} Z_{t-1} \Omega^{k} X_{\bullet},
$$

and so on. This does not imply, of course, that $\pi_{t, 1}^{\natural(k)} X_{\bullet} \cong \pi_{t, k+1}^{\natural} X_{\bullet}$.

We therefore see from (3.7) and (3.8) that the differential $d_{t, k}^{2}: E_{t, k}^{2} \rightarrow E_{t-2, k+1}^{2}$ may be identified with:

$$
\begin{aligned}
\pi_{t} \pi_{k} X \bullet \cong \pi_{t} \pi_{0}^{(k, 1)} X_{\bullet} \stackrel{\partial_{t, 0}^{(k, 1)}}{\longrightarrow} \Omega \pi_{t-2,0}^{\natural(k)} & X_{\bullet}=\pi_{t-2,1}^{\natural(k)} X_{\bullet} \\
& \stackrel{h_{t}}{\cong} \pi_{t-2} \pi_{1}^{(k, 1)} X \bullet \cong \pi_{t-2} \pi_{k+1} X \bullet
\end{aligned}
$$

Now by definition, $E_{t, k}^{3}$ fits into a commutative diagram

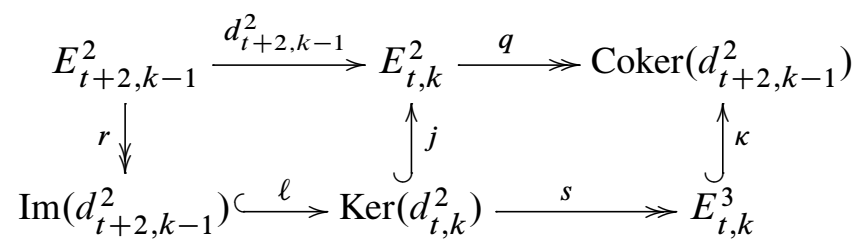

with exact rows, $\ell, j$ and $\kappa$ monic, and thus $E_{t, k}^{3} \cong \operatorname{Im}(q \circ j)$. 
From the exactness of (4.3) we see that

and

$$
\begin{aligned}
\operatorname{Coker}\left(d_{t+2, k-1}^{2}\right) & =\operatorname{Coker}\left(\partial_{t+2}^{(k-1,1)}\right)=\operatorname{Im}\left(s_{t+1}^{(k-1,1)}\right) \\
\operatorname{Ker}\left(d_{t, k}^{2}\right) & =\operatorname{Ker}\left(\partial_{t}^{(k, 1)}\right)=\operatorname{Im}\left(h_{t}^{(k, 1)}\right),
\end{aligned}
$$

so $E_{t, k}^{3}=\operatorname{Im}(q \circ j)$ is indeed the image of the map in (4.8).

4.11 Definition An $r$-truncated spectral sequence is one defined up to and including the $E^{r}$-term, together with the differential $d^{n}: E_{t, i}^{r} \rightarrow E_{t-r-1, t+r}^{r}$, but without requiring that $d^{r} \circ d^{r}=0$ (so the $E^{r+1}$-term is defined in terms of the $r$-truncated spectral sequence only if $d^{r} d^{r}=0$ ).

The main example is the $n$-truncation of an (ordinary) spectral sequence (such as that of a simplicial space). In this case we do have $d^{r} \circ d^{r}=0$, of course.

4.12 Corollary Any Reedy fibrant simplicial Postnikov 1-stem has a well-defined 2-truncated spiral spectral sequence. Moreover, if $\mathcal{Q}_{\bullet}=\mathcal{P}[1] X_{\bullet}$ for some simplicial space $X_{\bullet}$, this 2-truncated spectral sequence coincides with the 2-truncation of the Bousfield-Friedlander spectral sequence for $X_{\bullet}$.

In general, we have a less explicit description of the higher terms in the spiral spectral sequence:

4.13 Theorem For each $r \geq 0$, the $E^{r+2}$-term of the spiral spectral sequence for a simplicial space $X_{\bullet}$ is determined by the spiral $r$-system of $X_{\bullet}$. Moreover, for any $\alpha \in E_{t, i}^{r+1}$, we have $d_{t, i}^{r+1}(\alpha)=\beta \in E_{t-r-1, i+r}^{r+1}$ if and only if $\alpha$ and $\beta$ have representatives $\bar{a} \in \pi_{t} \pi_{i} X_{\bullet}$, and $\bar{b} \in \pi_{t-r-1} \pi_{i+r} X_{\bullet}$, respectively, such that:

$$
\left(s_{t-2,1}^{(i, r)}\right) \circ\left(s_{t-3,2}^{(i, r)}\right) \circ \cdots \circ\left(s_{t-r, r-1}^{(i, r)}\right) \circ\left(h_{t-r-1, r}^{(i, r)}\right)^{-1}(\bar{b})=\partial_{t, 0}^{(i, r)}(\bar{a})
$$

Proof We naturally identify $\pi_{t, k}^{\natural(i, r)} X$ • with $\pi_{t, k+s}^{\natural(i, r-s)} X$ • for $k \geq s$, and similarly for the maps in (4.3), so the spiral $(r-1)$-system embeds in the spiral $r$-system (with an index shift). 
Again we write out the $E^{1}$-term of the spiral exact couple, letting $P^{r}=P^{r} \Omega^{i} X_{\bullet}$ :

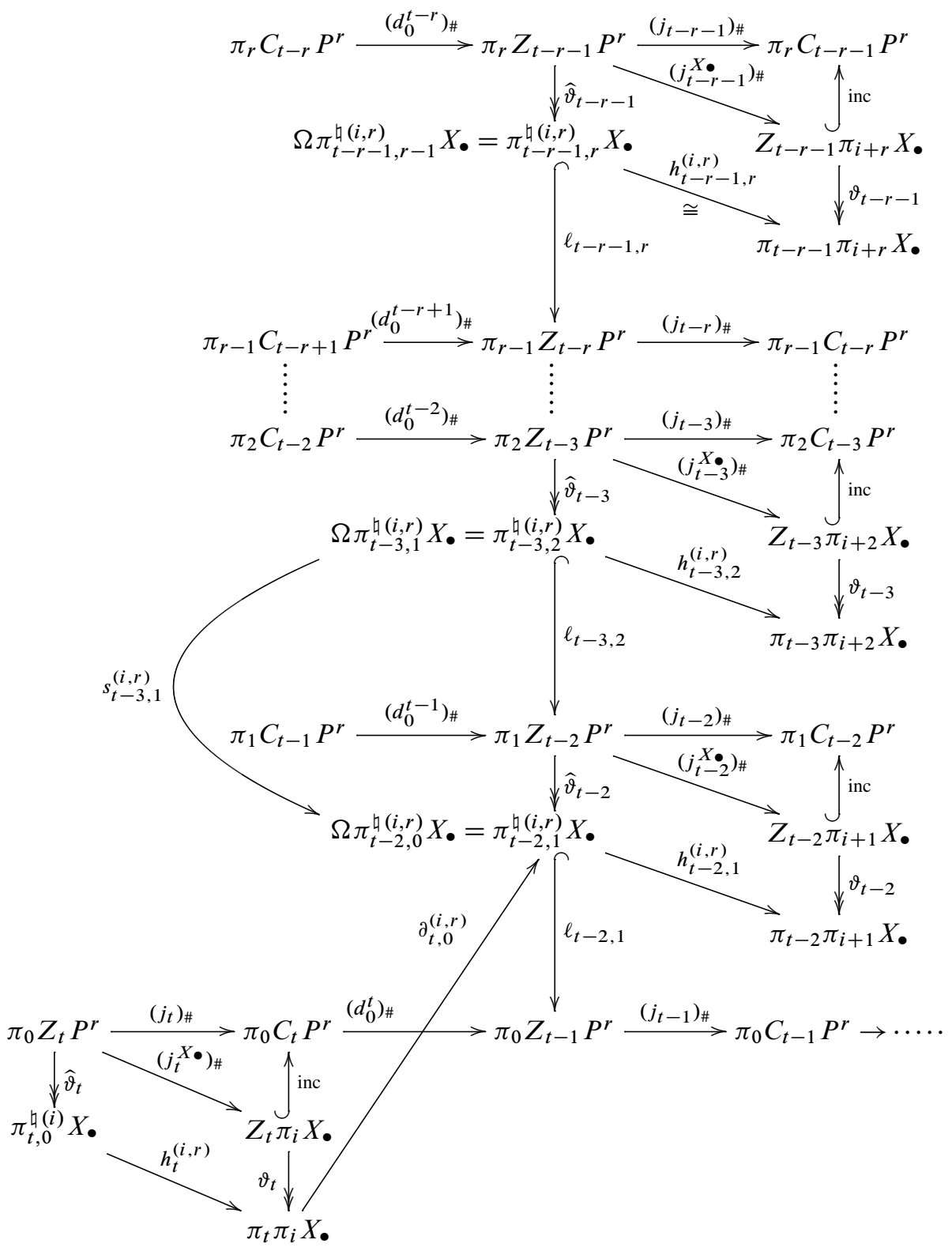

The differential $d_{t, i}^{r+1}: E_{t, i}^{r+1} \rightarrow E_{t-r-1, i+r}^{r+1}$ may then be described as a "relation" (cf [17, Section 3.1]) in the usual way:

Given a class $\alpha \in E_{t, i}^{r+1}$, choose a representative for it $a \in E_{t, i}^{1}=\pi_{0} C_{t} P^{r} \Omega^{i} X_{\bullet}$. Since it is a cycle for $d_{t, i}^{1}=\left(j_{t-1}\right)_{\#} \circ\left(d_{0}^{t}\right)_{\#}$, it lies in $Z_{t} \pi_{i} X_{\bullet}$ and thus represents an 
element $\bar{a} \in \pi_{t} \pi_{i} X_{\bullet}=E_{t, i}^{2}$. From the exactness of the middle row of (3.7) we see that

$$
\left(d_{0}^{t}\right)_{\#}(a) \in \operatorname{Ker}\left(\left(j_{t-1}\right)_{\#}\right)=\Omega \pi_{t-2,0}^{\natural(i, r)} X_{\bullet},
$$

and in fact $\left(d_{0}^{t}\right)_{\#}(a)$ represents $\partial_{t, 0}^{(i, r)}(\bar{a})$. Since $\widehat{\vartheta}_{t-2}$ is surjective, we can choose $e_{t-2} \in \pi_{1} Z_{t-2} P^{r} \Omega^{i} X_{\bullet}$ mapping to $\left(d_{0}^{t}\right)_{\#}(a)$. Because

$$
d_{t, i}^{2}(\bar{a})=h_{t-2,1}^{(i, r)} \circ \partial_{t, 0}^{(i, r)}(\bar{a}),
$$

as in the proof of Proposition 4.7 (though $h_{t-2,1}^{(i, r)}$ need no longer be an isomorphism!), we see that it is represented by $\left(j_{t-2}\right)_{*}\left(e_{t-2}\right)$. If $r=1$, we are done. Otherwise, we know that $d_{t, i}^{2}(\bar{a})=0$, so we can choose $e_{t-2}$ so that $\left(j_{t-2}\right)_{*}\left(e_{t-2}\right)=0$, using exactness of the third column of (3.7). Again this implies that

$$
e_{t-2} \in \operatorname{Ker}\left(\left(j_{t-2}\right)_{\#}\right)=\Omega \pi_{t-3,1}^{\natural(i, r)} X_{\bullet},
$$

and $d_{t, i}^{3}(\langle a\rangle)$ is represented by $h_{t-3,2}^{(i, r)}\left(e_{t-2}\right)$. Moreover, we see from (3.7) that

$$
s_{t-3,1}^{(i, r)}\left(e_{t-2}\right)=\partial_{t, 0}^{(i, r)}(\bar{a}),
$$

using the identification $\Omega \pi_{t-2,0}^{\natural(i, r)} X_{\bullet}=\pi_{t-2,1}^{\natural(i, r)} X_{\bullet}$.

Choosing a lift to $e_{t-3} \in \pi_{2} Z_{t-3} P^{r} \Omega^{i} X_{\bullet}$, we may assume that $\left(j_{t-3}\right)_{*}\left(e_{t-3}\right)=0$, so

$$
e_{t-3} \in \Omega \pi_{t-4,2}^{\natural(i, r)} X \bullet \quad \text { and } \quad s_{t-4,2}^{(i, r)}\left(e_{t-3}\right)=e_{t-2} .
$$

Continuing in this way, we finally reach

$$
e_{t-r-1} \in \Omega \pi_{t-r-1, r-1}^{\natural(i, r)} X \bullet \quad \text { with } \quad s_{t-r-2, r}^{(i, r)}\left(e_{t-r-1}\right)=e_{t-r},
$$

and so on, and see that $d_{t, i}^{r+1}(\langle a\rangle)$ is represented by $h_{t-r-1, r}^{(i, r)}\left(e_{t-r-1}\right)$. Since (as in the proof of Proposition 4.7) $h_{t-r-1, r}^{(i, r)}$ is an isomorphism, we deduce that $d_{t, i}^{r+1}(\alpha)$ is as in (4.14).

4.15 Remark From the exactness of (4.3) we have

$$
\operatorname{Im}\left(\partial_{t, 0}^{(i, r)}\right)=\operatorname{Ker}\left(s_{t-1,0}^{(i, r)}\right),
$$

so the image of $d_{t, i}^{r+1}$ as described in (4.14) is $\operatorname{Ker}\left(\sigma_{t, i}^{r+1}\right)$, where

$$
\sigma_{t, i}^{r+1}:=\left(s_{t-1,0}^{(i, r)}\right) \circ\left(s_{t-2,1}^{(i, r)}\right) \circ\left(s_{t-3,2}^{(i, r)}\right) \circ \cdots \circ\left(s_{t-r, r-1}^{(i, r)}\right) .
$$

Therefore, $E_{t+r-1, i+r}^{r+1}$ embeds naturally in $\operatorname{Im}\left(\sigma_{t, i}^{r+1}\right)$. 
4.16 Corollary Every Reedy fibrant simplicial Postnikov $r$-stem has a well-defined $(r+1)$-truncated spiral spectral sequence. If $\mathcal{Q}_{\bullet}=\mathcal{P}[r] X_{\bullet}$ for some simplicial space $X_{\bullet}$, this truncated spectral sequence coincides with the $(r+1)$-truncation of the Bousfield-Friedlander spectral sequence for $X_{\bullet}$.

Thus the bigraded homomorphism

$$
d^{r+1} \circ d^{r+1}: E_{t, i}^{r} \rightarrow E_{t-2 r-2, i+2 r}^{r+1} \quad(t \geq 2 r+2, i \geq 0)
$$

serves as the first obstruction to the realizability of the simplicial Postnikov $r$-stem $\mathcal{Q} \bullet$ by a simplicial space $X$.

\section{A cosimplicial version}

There are actually four variants of the above spectral sequence which we might consider, for a simplicial or cosimplicial object over simplicial or cosimplicial sets. The case of bicosimplicial sets is in principle strictly dual to that of bisimplicial sets, but because the category of cosimplicial sets has no (known) useful model category structure, we must restrict to bicosimplicial abelian groups - or equivalently, ordinary double complexes. Thus the main new case of interest is that of cosimplicial simplicial sets, or cosimplicial spaces.

5.1 The spectral sequence of a cosimplicial space If $X^{\bullet} \in c \mathcal{S}_{*}$ is a fibrant cosimplicial pointed space with total space Tot $X^{\bullet}$, there are various constructions for the homotopy spectral sequence of $X^{\bullet}$ :

(a) Using the tower of fibrations for $\left(\operatorname{Tot}_{n} X^{\bullet}\right)_{n=0}^{\infty}(\operatorname{cf}[15, X$, Section 6]).

(b) Using "relations" on the normalized cochains $N^{n} \pi_{t} X^{\bullet}:=\pi_{t} X^{n} \cap \operatorname{Ker}\left(s^{0}\right) \cap$ $\cdots \cap \operatorname{Ker}\left(s^{n-1}\right)$ (cf [17, Section 7]).

(c) Using a cofibration sequence dualizing (3.4) (cf [22, Section 3]).

Bousfield and Kan showed that the result is essentially unique (see [17]). Since the main ingredient needed for to define the spiral exact couple is the diagram (3.7), we use the first approach:

5.2 Definition For any Reedy fibrant cosimplicial pointed space $X^{\bullet} \in c \mathcal{S}_{*}$, consider the fibration sequence

$$
F_{n} X^{\bullet} \stackrel{j_{n}}{\longrightarrow} \operatorname{Tot}_{n} X^{\bullet} \stackrel{p_{n}}{\longrightarrow} \operatorname{Tot}_{n-1} X^{\bullet}
$$


where $\operatorname{Tot}_{n} X^{\bullet}:=\operatorname{map}_{c \mathcal{S}_{*}}\left(\operatorname{sk}_{n} \boldsymbol{\Delta}, X^{\bullet}\right)$ and the fibration $p_{n}$ is induced by the inclusion of cosimplicial spaces $\operatorname{sk}_{n-1} \Delta \hookrightarrow \operatorname{sk}_{n} \Delta$.

The cokernel of $\left(j_{n}\right)_{\#}: \pi_{*} F_{n} X^{\bullet} \hookrightarrow \pi_{*} \operatorname{Tot}_{n} X^{\bullet}$ is called the $n$-th natural (graded) cohomotopy group of $X^{\bullet}$, and denoted by $\pi_{\natural *}^{n} X^{\bullet}$.

5.4 Remark We may identify $F_{n} X^{\bullet}$ with the looped normalized cochain object $\Omega^{n} N^{n} X^{\bullet}$, where

$$
N^{n} X^{\bullet}:=X^{n} \cap \operatorname{Ker}\left(s^{0}\right) \cap \cdots \cap \operatorname{Ker}\left(s^{n-1}\right)
$$

and $\pi_{*} N^{n} X^{\bullet}$ with $N^{n} \pi_{*} X^{\bullet} \quad$ (see [15, X, Proposition 6.3]).

Moreover, the composite

$$
\begin{aligned}
& \pi_{*+1} \Omega^{n} N^{n} X^{\bullet} \cong \pi_{*+1} F_{n} X^{\bullet} \stackrel{\left(j_{n}\right)_{\#}}{\longrightarrow} \pi_{*+1} \operatorname{Tot}_{n} X^{\bullet} \\
& \stackrel{\partial_{n}}{\longrightarrow} \pi_{*} F_{n+1} X^{\bullet} \cong \pi_{*} \Omega^{n+1} N^{n+1} X^{\bullet}
\end{aligned}
$$

(where $\partial_{n}$ is the connecting homomorphism for the (5.3)), may then be identified with the differential

$$
\delta^{n}:=\sum_{i=0}^{n}(-1)^{i} d^{i}: N^{n} \pi_{*} X^{\bullet} \rightarrow N^{n+1} \pi_{*} X^{\bullet}
$$

for the normalized cochain complex $N^{*} \pi_{*} X^{\bullet}$, so that

$$
\operatorname{Ker}\left(\delta^{n}\right) / \operatorname{Coker}\left(\delta^{n+1}\right) \cong \pi^{n} \pi_{*} X^{\bullet}
$$

(cf [15, X, Section 7.2]).

5.8 Proposition For any pointed cosimplicial space $X^{\bullet}$ there is a natural spiral long exact sequence:

$$
\begin{aligned}
\cdots \rightarrow \Omega \pi_{\natural *}^{n-1} X^{\bullet} \stackrel{s^{n}}{\rightarrow} \pi_{\natural *}^{n} X^{\bullet} \stackrel{h^{n}}{\longrightarrow} \pi^{n} \pi_{*} X^{\bullet} \stackrel{\partial^{n}}{\longrightarrow} \Omega \pi_{\natural *}^{n-2} X^{\bullet} \\
\stackrel{s^{n-1}}{\longrightarrow} \pi_{\natural *}^{n-1} X^{\bullet} \rightarrow \cdots \rightarrow \pi_{\natural *}^{0} X^{\bullet} \stackrel{\cong}{\longrightarrow} \pi^{0} \pi_{*} X^{\bullet}
\end{aligned}
$$

Proof By choosing a fibrant replacement in the model category of cosimplicial simplicial sets defined in [15, X, Section 5], if necessary, we may assume that $X^{\bullet}$ is 
Reedy fibrant. We then obtain a commutative diagram as in (3.7) with exact rows and columns:

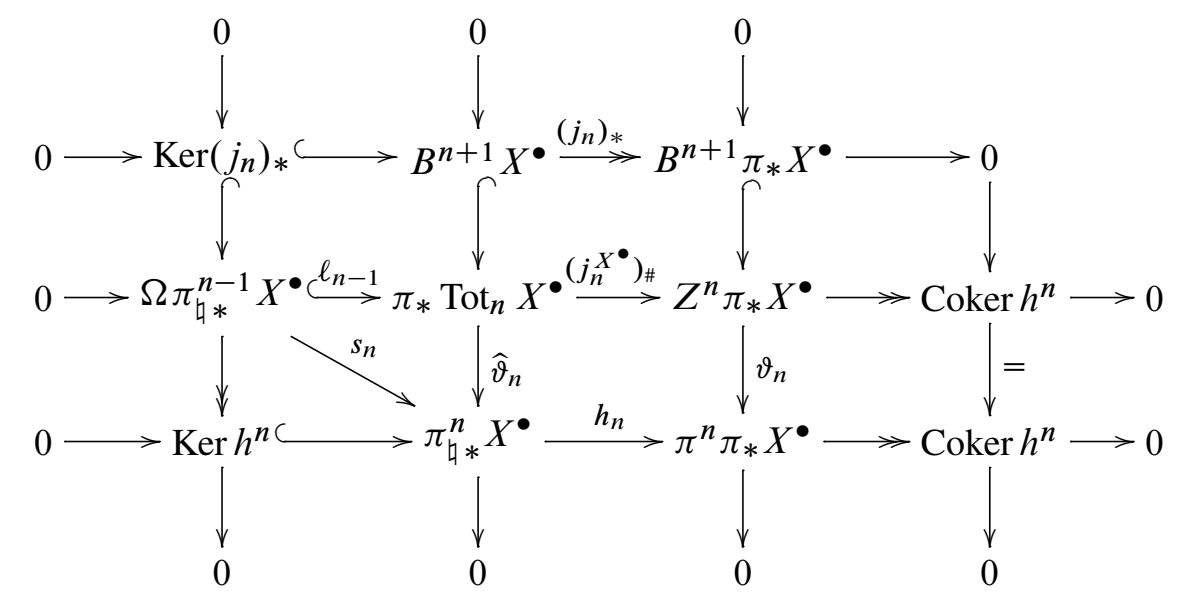

in which $B^{n+1} X^{\bullet}:=\operatorname{Im}\left(j_{n+1}\right)_{\#} \subseteq \pi_{*} \operatorname{Tot}_{n} X^{\bullet}$ and $B^{n+1} \pi_{*} X^{\bullet}:=\operatorname{Im}\left(\delta^{n+1}\right)=$ $\operatorname{Im}\left(\partial_{n+1} \circ\left(j_{n+1}\right)_{\#}\right)$ are the respective coboundary objects.

The construction of the maps $h^{n}, s^{n}$, and $\partial^{n}$, and the proof of the exactness of (5.9), are then precisely as in Section 3.6.

5.11 Definition The spiral $n-$ system of a pointed cosimplicial space $X^{\bullet} \in c \mathcal{S}_{*}$ is defined to be the collection of long exact sequences (5.9) for the Postnikov $n$-stem functor $\mathcal{P}[n]$ applied to $X^{\bullet}$, one for each $k$-window of $\mathcal{P}[n] X^{\bullet}$.

As in Definition 4.2, this may actually be defined for a cosimplicial Postnikov $n-$ stem $\mathcal{P}^{\bullet}$, not necessarily realizable as $\mathcal{P}^{\bullet}=\mathcal{P}[n] X^{\bullet}$.

By construction, the homotopy spectral sequence of a (fibrant) cosimplicial space $X^{\bullet}$, obtained as in 5.1, is associated to the spiral exact couple (5.9). The proofs of Proposition 4.7 and Theorem 4.13 use only the description of the spiral exact couple for $X \bullet$ derived from (5.10), so by using (5.10) instead we can prove their analogues in the cosimplicial case, and show:

5.12 Theorem The $E_{r+2}$-term of the homotopy spectral sequence for a cosimplicial space $X^{\bullet}$ is determined by the spiral $r$-system of $X^{\bullet}$.

An analogue of Corollary 4.16 also holds, as well as:

5.13 Proposition The differential $d_{2}^{t, i}: E_{2}^{t, i} \rightarrow E_{2}^{t+2, i+1}$ may be identified with $\partial_{(i, 1)}^{t}: \pi^{t} \pi_{i} X^{\bullet} \rightarrow \Omega \pi_{\natural(i)}^{t+2,0} X^{\bullet}$. 
5.14 Examples As noted in the introduction, many commonly used spectral sequences arise as the spiral spectral sequence of an appropriate (co)simplicial space, so Theorem 4.13 and Theorem 5.12 allow us to extract their $E^{r}-$ or $E_{r}$-terms from the appropriate spiral systems. For instance:

(a) Segal's homology spectral sequence (cf [23]), the van Kampen spectral sequence (cf [24]), and the Hurewicz spectral sequence (cf [9]) are constructed using bisimplicial sets.

(b) The unstable Adams spectral sequences of [13; 16] and [8, Section 4], Rector's version of the Eilenberg-Moore spectral sequence (cf [22]), and Anderson's generalization of the latter (cf [2]) are all associated to cosimplicial spaces.

(c) The usual construction of the stable Adams spectral sequence for $\pi_{*}^{s} X \otimes \mathbb{Z} / p$ (cf [1, Section 3]) uses a tower of (co)fibrations, rather than a cosimplicial space, but when $X$ is finite dimensional, it agrees in a range with the unstable version for $\Sigma^{N} X$, so Theorem 5.12 applies stably, too.

\section{References}

[1] J F Adams, On the structure and applications of the Steenrod algebra, Comment. Math. Helv. 32 (1958) 180-214 MR0096219

[2] DW Anderson, A generalization of the Eilenberg-Moore spectral sequence, Bull. Amer. Math. Soc. 78 (1972) 784-786 MR0310889

[3] H-J Baues, Homotopy type and homology, Oxford Math. Monogr., Oxford Science Publ., The Clarendon Press, Oxford Univ. Press, New York (1996) MR1404516

[4] H-J Baues, D Blanc, Comparing cohomology obstructions, to appear in J. Pure Appl. Alg. arXiv: 1008.1712

[5] H-J Baues, D Blanc, Higher order derived functors and spectral sequence, Preprint (2010)

[6] H-J Baues, M Jibladze, Secondary derived functors and the Adams spectral sequence, Topology 45 (2006) 295-324 MR2193337

[7] H-J Baues, F Muro, Secondary homotopy groups, Forum Math. 20 (2008) 631-677 MR2431499

[8] M Bendersky, E B Curtis, H R Miller, The unstable Adams spectral sequence for generalized homology, Topology 17 (1978) 229-248 MR508887

[9] D Blanc, A Hurewicz spectral sequence for homology, Trans. Amer. Math. Soc. 318 (1990) 335-354 MR956029

[10] D Blanc, CW simplicial resolutions of spaces with an application to loop spaces, Topology Appl. 100 (2000) 151-175 MR1733041 
[11] A K Bousfield, Homotopy spectral sequences and obstructions, Israel J. Math. 66 (1989) 54-104 MR1017155

[12] A K Bousfield, Cosimplicial resolutions and homotopy spectral sequences in model categories, Geom. Topol. 7 (2003) 1001-1053 MR2026537

[13] A K Bousfield, E B Curtis, D M Kan, D G Quillen, D L Rector, J W Schlesinger, The mod-p lower central series and the Adams spectral sequence, Topology 5 (1966) 331-342 MR0199862

[14] A K Bousfield, E M Friedlander, Homotopy theory of $\Gamma$-spaces, spectra, and bisimplicial sets, from: "Geometric applications of homotopy theory (Proc. Conf., Evanston, Ill., 1977), II”, (M G Barratt, ME Mahowald, editors), Lecture Notes in Math. 658, Springer, Berlin (1978) 80-130 MR513569

[15] A K Bousfield, D M Kan, Homotopy limits, completions and localizations, Lecture Notes in Math. 304, Springer, Berlin (1972) MR0365573

[16] A K Bousfield, D M Kan, The homotopy spectral sequence of a space with coefficients in a ring., Topology 11 (1972) 79-106 MR0283801

[17] A K Bousfield, D M Kan, A second quadrant homotopy spectral sequence, Trans. Amer. Math. Soc. 177 (1973) 305-318 MR0372859

[18] W G Dwyer, D M Kan, C R Stover, An $E^{2}$ model category structure for pointed simplicial spaces, J. Pure Appl. Algebra 90 (1993) 137-152 MR1250765

[19] W G Dwyer, D M Kan, CR Stover, The bigraded homotopy groups $\pi_{i, j} X$ of a pointed simplicial space X, J. Pure Appl. Algebra 103 (1995) 167-188 MR1358761

[20] PS Hirschhorn, Model categories and their localizations, Math. Surveys and Monogr. 99, Amer. Math. Soc. (2003) MR1944041

[21] D G Quillen, Spectral sequences of a double semi-simplicial group, Topology 5 (1966) 155-157 MR0195097

[22] D L Rector, Steenrod operations in the Eilenberg-Moore spectral sequence, Comment. Math. Helv. 45 (1970) 540-552 MR0278310

[23] G Segal, Classifying spaces and spectral sequences, Inst. Hautes Études Sci. Publ. Math. (1968) 105-112 MR0232393

[24] C R Stover, A van Kampen spectral sequence for higher homotopy groups, Topology 29 (1990) 9-26 MR1046622

Max-Planck-Institut für Mathematik

Vivatsgasse 7, PO Box 7280, D-53111 Bonn, Germany

Department of Mathematics, University of Haifa

31905 Haifa, Israel

baues@mpim-bonn.mpg.de, blanc@math.haifa.ac.il

Received: 1 April 2010 Revised: 10 August 2010 